\title{
Use of Plasmodium falciparum culture- adapted field isolates for in vitro exflagellation-blocking assay
}

\author{
Louis-Jérôme Leba ${ }^{1,2}$, Lise Musset ${ }^{1}$, Stéphane Pelleau', Yannick Estevez ${ }^{2}$, Caroline Birer ${ }^{2}$, Sébastien Briolant ${ }^{1,3,4}$, \\ Benoit Witkowski ${ }^{5}$, Didier Ménard ${ }^{5}$, Michael J Delves ${ }^{6}$, Eric Legrand ${ }^{1}$, Christophe Duplais ${ }^{2 \dagger}$ and Jean Popovici ${ }^{1,5^{*}+}$
}

\begin{abstract}
Background: A major requirement for malaria elimination is the development of transmission-blocking interventions. In vitro transmission-blocking bioassays currently mostly rely on the use of very few Plasmodium falciparum reference laboratory strains isolated decades ago. To fill a piece of the gap between laboratory experimental models and natural systems, the purpose of this work was to determine if culture-adapted field isolates of $P$. falciparum are suitable for in vitro transmission-blocking bioassays targeting functional maturity of male gametocytes: exflagellation.

Methods: Plasmodium falciparum isolates were adapted to in vitro culture before being used for in vitro gametocyte production. Maturation was assessed by microscopic observation of gametocyte morphology over time of culture and the functional viability of male gametocytes was assessed by microscopic counting of exflagellating gametocytes. Suitability for in vitro exflagellation-blocking bioassays was determined using dihydroartemisinin and methylene blue.

Results: In vitro gametocyte production was achieved using two isolates from French Guiana and two isolates from Cambodia. Functional maturity of male gametocytes was assessed by exflagellation observations and all four isolates could be used in exflagellation-blocking bioassays with adequate response to methylene blue and dihydroartemisinin.

Conclusion: This work shows that in vitro culture-adapted P. falciparum field isolates of different genetic background, from South America and Southeast Asia, can successfully be used for bioassays targeting the male gametocyte to gamete transition, exflagellation.
\end{abstract}

\section{Background}

Among the actions required for malaria elimination, blocking the transmission of Plasmodium parasites from human to mosquitoes is critical [1]. Passage through the vector is an obligatory step for the parasite to continue its life cycle and it relies exclusively on the most mature forms of the sexual stages, stage $V$ gametocytes. Mosquito feeding assays remain the gold standard to evaluate transmission-blocking strategies but require resourceintensive techniques. To circumvent these technical difficulties, several in vitro transmission-blocking bioassays

\footnotetext{
* Correspondence: jean.o.popovici@gmail.com

${ }^{\dagger}$ Equal contributors

'Laboratoire de Parasitologie, Institut Pasteur de la Guyane, Cayenne, French Guiana, France

${ }^{5}$ Malaria Molecular Epidemiology Unit, Institut Pasteur du Cambodge, Phnom Penh, Cambodia

Full list of author information is available at the end of the article
}

targeting the sexual stages of the parasite have been described with different endpoints and various interpretative values $[2,3]$. Although the outputs of these in vitro assays are difficult to transpose in vivo, the most clinically relevant assay should be able to evaluate the ability of a compound to either kill stage $\mathrm{V}$ gametocytes (viability assay) or inhibit their ability to differentiate into later mosquito stages (functional maturity assay). It is possible to induce in vitro gamete formation from Plasmodium falciparum male and female gametocytes where male gametocytes undergo major differentiation leading to the production of eight mobile gametes, by a process called exflagellation, which is easily observed visually.

Currently, the majority of $P$. falciparum in vitro transmission-blocking studies use a handful of reference laboratory strains, isolated decades ago. While these strains are useful to normalize high throughput screenings, results should be verified on natural parasites that 
have been selected after years of multiple drug exposures and hence are likely to display differential drug responses compared to reference strains. In addition, it is well known that gametocyte production capacity is lost over time of in vitro culture [2, 4]. Laboratories must therefore rely on precious stocks of cryopreserved isolates with minimal passage since in culture. Adaptation of $P$. falciparum isolates from patients to in vitro blood-stage culture is routinely performed. Studies describing the use of field isolates for in vitro gametocyte cultures have been published in the early 1980s and have led to the selection of the current reference strains [4-8]. Nowadays using circulating parasites after culture adaptation for transmissionblocking assay is rarely performed. Some studies have shown that they can be used in gametocyte viability assay [9] or for experimental infections of mosquitoes [10], however their use for exflagellation-blocking bioassays, reporting in vitro functional maturity of gametocytes has not been reported yet. A requirement for their use in such assay is to be able to produce functional gametocytes in high enough number to be meaningful in a bioassay.

The objective of the work presented here was therefore to determine if culture-adapted field isolates could be used for recently developed in vitro exflagellationblocking bioassays [11].

\section{Methods}

\section{Reference strain and field isolates}

The P. falciparum South American chloroquine-resistant strain 7G8 (MRA-926) has been obtained from the MR4. Plasmodium falciparum isolates were collected from monoinfected patients seeking treatment in 2013 in French Guiana (Q206 and Q188) and in 2014 in Cambodia (6831 and 6836).

\section{In vitro culture adaptation}

Culture adaptation of isolates was performed using standard protocols $[12,13]$. Briefly, after removal of plasma, the red blood cell $(\mathrm{RBC})$ pellet was washed three times in RPMI 1640 supplemented with gentamicin (Gibco-Life Technologies SAS, France) and placed in culture medium (RPMI 1640, $0.5 \%$ AlbuMAX II (Gibco-Life Technologies SAS, France), $2 \%$ decomplemented human plasma) at $4 \%$ haematocrit at $37{ }^{\circ} \mathrm{C}$ in 5 or $10 \% \mathrm{O}_{2}, 5 \% \mathrm{CO}_{2}$, rest of $\mathrm{N}_{2}$ atmosphere. Parasitaemia was checked daily and kept below $2 \%$ by dilutions with fresh RBC and medium. Field isolates were considered adapted to in vitro conditions after three weeks of uninterrupted culture. After culture adaptation, asexual blood-stage sensitivity to anti-malarials was determined using the $\left[{ }^{3} \mathrm{H}\right]$-hypoxanthine incorporation assay [14].

\section{In vitro gametocyte production and maturation}

Gametocyte cultures were performed following published protocols $[2,11]$. Briefly, asexual cultures with a parasitaemia of $\sim 5 \%$ were used to seed gametocyte cultures at $0.5-1 \%$ parasitaemia and $4 \%$ haematocrit under 5 or $10 \% \mathrm{O}_{2}, 5 \% \mathrm{CO}_{2}$, rest of $\mathrm{N}_{2}$ atmosphere. Culture medium (RPMI medium with $25 \mathrm{mM}$ HEPES, $50 \mathrm{mg} / \mathrm{L}$ hypoxanthine, $2 \mathrm{~g} / \mathrm{L}$ sodium bicarbonate, $10 \%$ human serum) was replaced daily but without any further addition of RBCs and critically, temperature was maintained at all time at $37^{\circ} \mathrm{C}$.

Gametocytogenesis was evaluated morphologically using Giemsa-stained blood films. Stage V male gametocyte functional maturity was assessed by observation of exflagellation in wet preparation under bright-field microscope. A $30-\mu \mathrm{L}$ aliquot of gametocyte culture was briefly centrifuged, the cell pellet was resuspended in $15 \mu \mathrm{L}$ of ookinete medium (RPMI medium with $25 \mathrm{mM}$ HEPES, $50 \mathrm{mg} / \mathrm{L}$ hypoxanthine, $2 \mathrm{~g} / \mathrm{L}$ sodium bicarbonate, $100 \mu \mathrm{M}$ xanthurenic acid, $10 \%$ human serum) and then introduced into a chamber of a FastRead disposable haemocytometer slide (Immune Systems). Exflagellation centres were observed at $10 \times$ or $20 \times$ magnification.

\section{Exflagellation-blocking bioassay}

Exflagellation-blocking assays were performed according to published protocols [11]. Assays were performed using gametocyte cultures providing high enough exflagellation centres for meaningful measures ( $>30$ in five $10 \times$ microscopy fields).

To show the suitability of field isolates for exflagellationblocking assays, the activity of $1 \mu \mathrm{M}$ of dihydroartemisinin (DHA), methylene blue (MeBlue) and chloroquine (CQ) was evaluated. Assays were carried out in $1.5-\mathrm{mL}$ tubes containing $170 \mu \mathrm{L}$ of gametocyte culture medium with $1 \mu \mathrm{M}$ of drugs dissolved in either DMSO or methanol. Thirty $\mu \mathrm{L}$ of stage $\mathrm{V}$ mature gametocyte was dispensed into each assay tube. Tubes were then placed into a $37^{\circ} \mathrm{C}$ incubator. After $24 \mathrm{~h}$, exflagellation was induced by temperature drop to $25{ }^{\circ} \mathrm{C}$ and replacing culture medium with ookinete medium. The number of exflagellation centres were recorded and compared to controls (DMSO or methanol). Activity was expressed as percentage of exflagellation inhibition compared to controls.

\section{Results}

The protocols were first optimized by using the South American isolates prior to being verified with the Southeast Asian isolates. Gametocyte development was straightforward at the first attempt. Initially however, although stage $\mathrm{V}$ gametocytes were clearly identified in the cultures, no exflagellation was observed. This suggests that bioassays with a read-out based only on morphological development of gametocytes do not report on their onward functional viability, therefore they may be underpowered. As already observed by others $[2,4]$, the age of uninfected $R B C$ when initiating the gametocyte 
culture was a critical factor for successful maturation: RBC stored at $4{ }^{\circ} \mathrm{C}$ more than $\sim$ two to 3 days rapidly compromise maturation. Additionally, variability in human serum was found also to be a critical point for gametocyte maturation, so pools from different donors of serum were used when possible. Using fresh uninfected RBC, fully mature gametocytes capable of exflagellation were generated for all isolates. For all isolates and 7G8, gametocyte development time was similar and maturity peaked at $~ 16-18$ days of culture, slightly longer than what is usually reported for 3D7, 12-14 days (Fig. 1) [2, 11].

It is important to note that all the experiments using 7G8 and the South American isolates were performed in a different laboratory (Institut Pasteur de la Guyane, Cayenne) than those using the Cambodian isolates (Institut Pasteur du Cambodge, Phnom Penh) showing good reproducibility in the protocols that can be easily implemented in laboratories doing P. falciparum in vitro culture adaptation.

Exflagellation is a time-dependent process [15]. Within $20 \mathrm{~min}$ after induction, for all field isolates and the reference strain 7G8, a plateau is reached allowing consistent measurements for $\sim 10 \mathrm{~min}$ (Fig. 2). This is similar to what has previously been observed with 3D7 [11].

Although the patterns of gametocyte development and time for exflagellation are similar for all isolates and laboratory strains, there are variations in the amount of exflagellation centres from one isolate to another (see Additional file 1) as already observed by others [4]. For example, in French Guiana, the isolate Q206 consistently gave higher number of exflagellation than Q188. Nevertheless, for all the isolates, using gametocyte

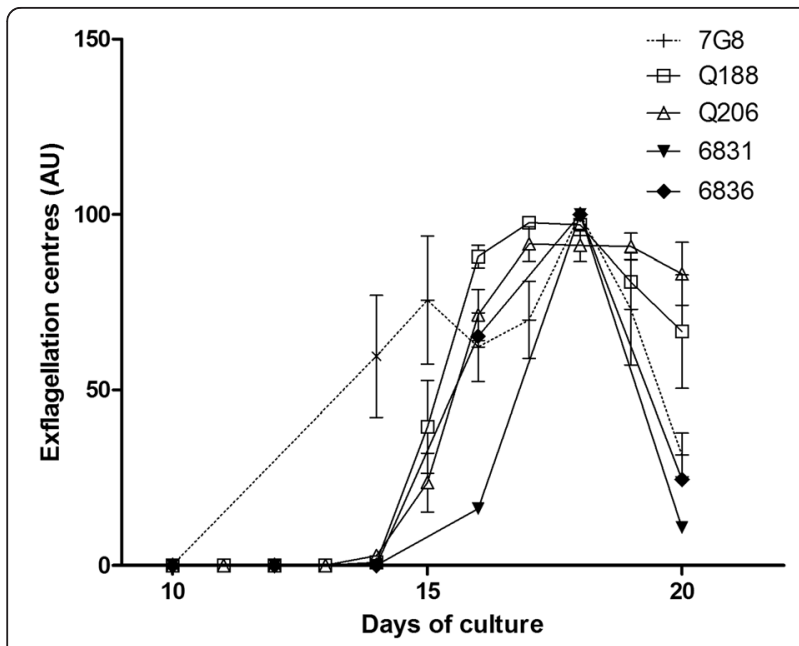

Fig. 1 Exflagellation levels over days of gametocyte culture (arbitrary unit). For each independent culture, exflagellation centres values are normalized to the highest value of the culture (data are presented in Additional file 1). The mean and SEM of three cultures is shown for 7 G8 and the two French Guiana strains (Q188 and Q206) while a single culture for each Cambodian isolate is represented (6831 and 6836)

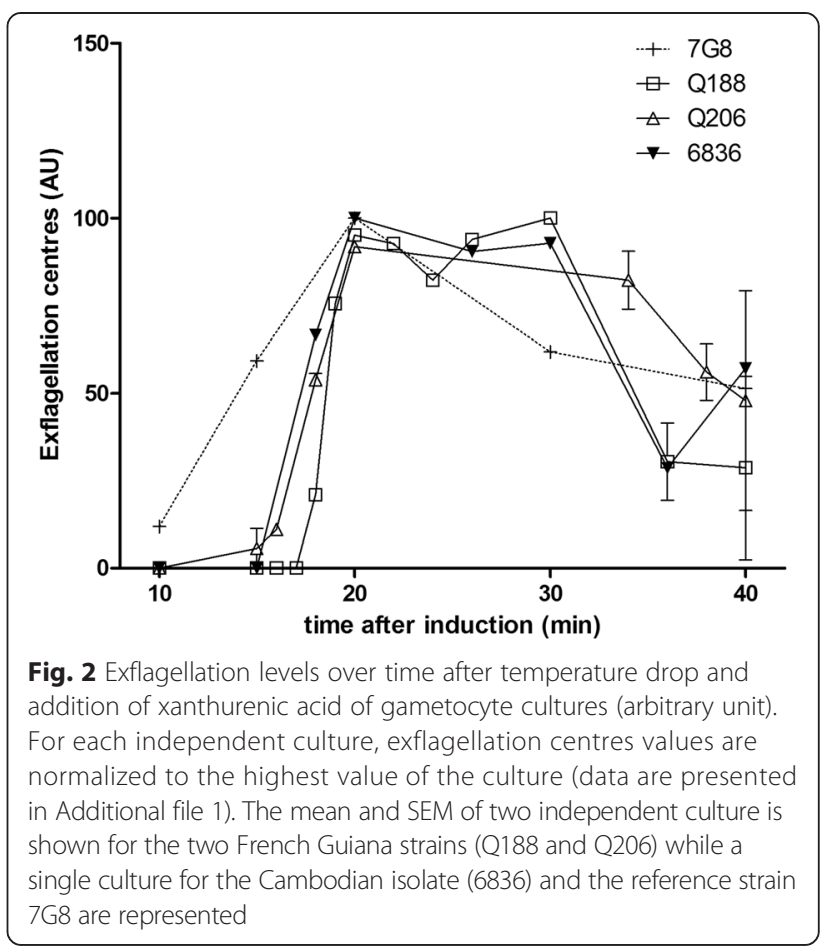

cultures at maturity, the numbers of exflagellation centres obtained were high enough to allow significant measures in exflagellation-blocking bioassay (from $\sim 35$ to $>100$, see Additional file 1).

Based on those observations, a previously described exflagellation-blocking protocol was adapted to the four field isolates and 7G8 [11]. Exflagellation centres were recorded between 20 and $30 \mathrm{~min}$ after induction of 18 days-old gametocyte cultures. As a proof-of-concept, the activity of DHA and MeBlue was evaluated as both compounds have been previously reported to block exflagellation of 3D7 [11]. As negative control, CQ was used on a sub-set of the strains [16]. Mature gametocytes were incubated $24 \mathrm{~h}$ in presence of $1 \mu \mathrm{M}$ of each drug before exflagellation was induced. All isolates responded similarly to the compounds with $1 \mu \mathrm{M}$ DHA giving 78-99 \% inhibition and consistently near total inhibition with $1 \mu \mathrm{M}$ of MeBlue (Table 1). As expected, CQ had no effect on exflagellation in any isolate tested.

Table 1 Exflagellation-blocking activity a of dihydroartemisinin (DHA), methylene blue (MeBlue) and chloroquine (CQ) against Plasmodium falciparum field isolates and the $7 \mathrm{G} 8$ reference strain

\begin{tabular}{llllll}
\hline & $7 G 8$ & Q188 & Q206 & 6831 & 6836 \\
\hline DHA & $99 \pm 1.3$ & $90 \pm 8.3$ & $78 \pm 7.2$ & $84 \pm 12.2$ & $79 \pm 11.6$ \\
MeBlue & 100 & 100 & $99 \pm 1.7$ & 100 & $99 \pm 2.1$ \\
CQ & $-1 \pm 10.8$ & ND & $5.7 \pm 10.2$ & ND & $7.3 \pm 19.2$
\end{tabular}

${ }^{\mathrm{a}}$ Expressed as a percentage of inhibition compared to the drug-free control (mean $\pm \mathrm{SEM}, n=3$ ) 


\section{Conclusion}

This work reports that freshly culture-adapted field isolates, from various geographical origins and genetic background can produce functionally mature gametocytes in vitro in high enough numbers to be successfully used in exflagellation-blocking bioassays. Moreover, the assay was sensitive enough to observe inter-strain differences, which might be of significant interest for comprehension of malaria transmission in the field. Indeed, the Q188 isolate is chloroquine-sensitive $\left(\mathrm{IC}_{50}=34.93\right.$ $\mathrm{nM} \pm 1.91)$ and consistently produced fewer gametocytes in vitro than the Q206 isolate that is chloroquineresistant $\left(\mathrm{IC}_{50}=483.36 \mathrm{nM} \pm 61.25\right)$. Of course, given the low number of isolates, no conclusion can be drawn based on this work and further studies are required to evaluate a possible link between chemosensitivity and in vitro gametocytogenesis. In addition, at least one cryopreservation cycle did not impair the capacity of the field isolates in producing mature gametocytes. However, for how long in culture this capacity will be maintained before being lost, as expected remains to be evaluated. Finally, the infectivity to mosquitoes of the mature gametocytes should be assessed to transpose the in vitro results to an in vivo model of transmission. Capacity building for experimental infections of mosquitoes is currently being undergone in Institut Pasteur, both in French Guiana and in Cambodia, and will allow such experiments in a near future.

\section{Additional file}

Additional file 1: Absolute and normalized exflagellation values.

Dataset including exflagellation values over days of culture and exflagellation values over time after induction.

\section{Competing interests}

The authors have declared that they have no competing interests.

\section{Authors' contributions \\ LUL carried out gametocyte culture, exflagellation experiments and analysed the data. LM and SP supplied culture-adapted field isolates, provided support in gametocyte culture and analysed the data. YE and CB helped with the exflagellation experiments. SB provided technical support. MD contributed to the protocol design and data analysis. BW, DM, and EL supplied culture- adapted field isolates. CD and JP supervised this work, conceived the study, its design and its coordination. JP wrote the manuscript. All authors read and approved the final manuscript.}

\section{Acknowledgements}

This work was supported by a European commission REGPOT-CT-2011-285837STRonGer Grant within the FP7, by an Institut Pasteur ACIP (A10-2013) Grant and by Investissement d'Avenir Grant of the ANR (CEBA: ANR-10-LABX-25-01). We thank MR4 for providing Plasmodium falciparum 7G8, MRA-926 malaria parasites deposited by Thomas Wellems.

\section{Author details}

'Laboratoire de Parasitologie, Institut Pasteur de la Guyane, Cayenne, French Guiana, France. ${ }^{2}$ Laboratoire ECOFOG (Ecology of Guiana Forests) UMR8172 CNRS-INRA-CIRAD-AgroParisTech-Université des Antilles-Université de la Guyane, French Guiana, France. ${ }^{3}$ Direction Interarmées du Service de Santé en Guyane, Cayenne, French Guiana, France. ${ }^{4}$ Institut de Recherche Biomédicale des Armées, Brétigny sur Orge, France. ${ }^{5}$ Malaria Molecular Epidemiology Unit, Institut Pasteur du Cambodge, Phnom Penh, Cambodia. ${ }^{6}$ Department of Life Sciences, Imperial College, London, UK.

Received: 14 April 2015 Accepted: 27 May 2015

Published online: 04 June 2015

\section{References}

1. Alonso PL, Brown G, Arevalo-Herrera M, Binka F, Chitnis C, Collins F, et al. $A$ research agenda to underpin malaria eradication. PLoS Med. 2011;8:e1000406. doi:10.1371/journal.pmed.1000406.

2. Blagborough A, Delves M, Ramakrishnan C, Lal K, Butcher G, Sinden R. Assessing transmission blockade in Plasmodium spp. In: Ménard R, editor. Malaria. Methods in Molecular Biology. New York: Humana Press; 2013. p. 577-600.

3. Sinden RE, Blagborough AM, Churcher $\mathrm{T}$, Ramakrishnan $\mathrm{C}$, Biswas $\mathrm{S}$, Delves MJ. The design and interpretation of laboratory assays measuring mosquito transmission of Plasmodium. Trends Parasitol. 2012;28:457-65. doi:10.1016/j.pt.2012.07.005

4. Ponnudurai T, Meuwissen JHET, Leeuwenberg ADEM, Verhave JP, Lensen AHW. The production of mature gametocytes of Plasmodium falciparum in continuous cultures of different isolates infective to mosquitoes. Trans R Soc Trop Med Hyg. 1982;76:242-50. doi:10.1016/0035-9203(82)90289-9.

5. Ifediba T, Vanderberg JP. Complete in vitro maturation of Plasmodium falciparum gametocytes. Nature. 1981;294:364-6.

6. Campbell CC, Chin W, Collins WE, Moss DM. Infection of Anopheles freeborni by gametocytes of cultured Plasmodium falciparum. Trans R Soc Trop Med Hyg. 1980;74:668-9. http://dx.doi.org/10.1016/0035-9203(80)90163-7.

7. Ponnudurai T, Verhave JP, Meuwissen JHET. Mosquito transmission of cultured Plasmodium falciparum. Trans R Soc Trop Med Hyg. 1982;76:278-9. http://dx.doi.org/10.1016/0035-9203(82)90298-X.

8. Burkot TR, Williams JL, Schneider I. Infectivity to mosquitoes of Plasmodium falciparum clones grown in vitro from the same isolate. Trans R Soc Trop Med Hyg. 1984;78:339-41. doi:10.1016/0035-9203(84)90114-7.

9. Gebru T, Mordmüller B, Held J. Effect of fluorescent dyes on in-vitro differentiated, late-stage Plasmodium falciparum gametocytes. Antimicrob Agents Chemother. 2014;58:7398-404. doi:10.1128/aac.03772-14.

10. Chotivanich K, Sattabongkot J, Udomsangpetch R, Looareesuwan S, Day NPJ, Coleman RE, et al. Transmission-blocking activities of quinine, primaquine, and artesunate. Antimicrob Agents Chemother. 2006;50:1927-30. doi:10.1128/aac.01472-05.

11. Delves MJ, Ruecker A, Straschil U, Lelièvre J, Marques S, López-Barragán MJ, et al. Male and female Plasmodium falciparum mature gametocytes show different responses to antimalarial drugs. Antimicrob Agents Chemother. 2013;57:3268-74. doi:10.1128/aac.00325-13

12. Witkowski B, Khim N, Chim P, Kim S, Ke S, Kloeung N, et al. Reduced artemisinin susceptibility of Plasmodium falciparum ring stages in Western Cambodia. Antimicrob Agents Chemother. 2013;57:914-23. doi:10.1128/aac.01868-12

13. Trager W, Jensen J. Human malaria parasites in continuous culture. Science. 1976;193:673-5. doi:10.1126/science.781840.

14. Desjardins RE, Canfield CJ, Haynes JD, Chulay JD. Quantitative assessment of antimalarial activity in vitro by a semiautomated microdilution technique. Antimicrob Agents Chemother. 1979;16:710-8. doi:10.1128/aac.16.6.710.

15. Bhattacharyya MK, Kumar N. Effect of xanthurenic acid on infectivity of Plasmodium falciparum to Anopheles stephensi. Int J Parasit. 2001;31:1129-33. http://dx.doi.org/10.1016/S0020-7519(01)00222-3.

16. Delves M, Plouffe D, Scheurer C, Meister S, Wittlin S, Winzeler EA, et al. The activities of current antimalarial drugs on the life cycle stages of Plasmodium: a comparative study with human and rodent parasites. PLoS Med. 2012;9:e1001169. doi:10.1371/journal.pmed.1001169. 